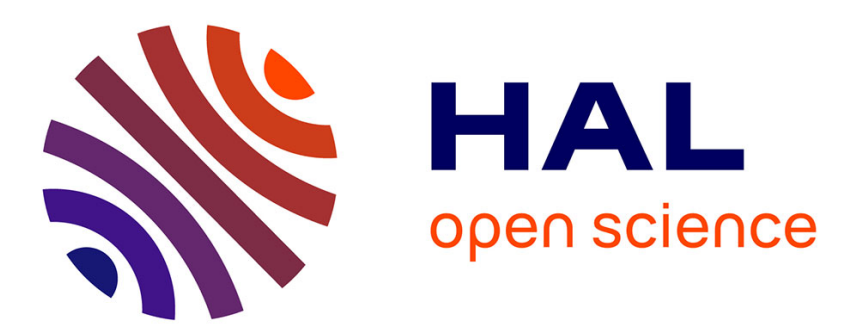

\title{
Experimental characterization and theoretical analysis of cell tip oscillations in directional solidification
}

\author{
J. Pereda, Fatima L. Mota, J.-M. Debierre, B. Billia, R. Trivedi, A. Karma,
} N. Bergeon

\section{- To cite this version:}

J. Pereda, Fatima L. Mota, J.-M. Debierre, B. Billia, R. Trivedi, et al.. Experimental characterization and theoretical analysis of cell tip oscillations in directional solidification. Physical Review E , 2020, 102 (3), 10.1103/PhysRevE.102.032804 . hal-02951894

\section{HAL Id: hal-02951894 \\ https://hal.science/hal-02951894}

Submitted on 29 Sep 2020

HAL is a multi-disciplinary open access archive for the deposit and dissemination of scientific research documents, whether they are published or not. The documents may come from teaching and research institutions in France or abroad, or from public or private research centers.
L'archive ouverte pluridisciplinaire HAL, est destinée au dépôt et à la diffusion de documents scientifiques de niveau recherche, publiés ou non, émanant des établissements d'enseignement et de recherche français ou étrangers, des laboratoires publics ou privés. 


\title{
Experimental characterization and theoretical analysis of cell tip oscillations in directional solidification
}

\author{
J. Pereda, ${ }^{1}$ F. L. Mota $\odot,{ }^{1},{ }^{*}$ J.-M. Debierre,${ }^{1}$ B. Billia,${ }^{1}$ R. Trivedi,${ }^{2}$ A. Karma, ${ }^{3}$ and N. Bergeon ${ }^{1}$ \\ ${ }^{1}$ Aix-Marseille Université, CNRS, Université de Toulon, IM2NP UMR 7334, 13397 Marseille, France \\ ${ }^{2}$ Department of Materials Science and Engineering, Iowa State University, Ames, Iowa 50010, USA \\ ${ }^{3}$ Department of Physics and Center for Interdisciplinary Research on Complex Systems, Northeastern University, \\ Boston, Massachusetts 02115, USA
}

(Received 15 July 2020; accepted 8 September 2020; published xxxxxxxxxx)

\begin{abstract}
Experiments performed in DECLIC-DSI on board the International Space Station evidenced oscillatory modes during the directional solidification of a bulk sample of succinonitrile-based transparent alloy. The interferometric data acquired during a reference experiment, $V_{p}=1 \mu \mathrm{m} / \mathrm{s}$ and $G=19 \mathrm{~K} / \mathrm{cm}$, allowed us to reconstruct the cell shape and thus measure the cell tip position, radius, and growth velocity evolution, in order to quantify the dynamics of the oscillating cells. This study completes our previous reports [Bergeon et al., Phys. Rev. Lett. 110, 226102 (2013); Tourret et al., Phys. Rev. E 92, 042401 (2015); Pereda et al., Phys. Rev. E 95, 012803 (2017)] with, to our knowledge, the first complete monitoring of the geometric cell tip characteristics variations in bulk samples. The evolution of the shape, velocity, and position of the tip of the oscillating cells is associated with an evolution of the concentration field, inaccessible experimentally but mediating the diffusive interactions between the cells. The experimental results are supported by $3 \mathrm{D}$ phase-field simulations which evidence the existence of transversal solute fluxes between neighboring cells that play a fundamental role in the oscillation dynamics. The dynamics of oscillation of an individual cell is analyzed using a theoretical model based on classical equations of solidification through the calculation of the phase relationships between oscillation of the different tip characteristics.
\end{abstract}

DOI: 10.1103/PhysRevE.00.002800

\section{INTRODUCTION}

In a wide range of scientific fields, pattern formation, a quite complex process occurring in highly nonlinear growth regimes, is of paramount importance [1]. Nonequilibrium interface patterns can be obtained by directional solidification of binary alloys. These patterns have been widely studied because they are analogous to patterns formed in other fields (combustion, fluid dynamics, geology, biology, etc.) and because of their metallurgical relevance [2-4]. During directional solidification, the crucible containing a binary alloy of nominal solute concentration $C_{0}$ is placed inside a furnace that imposes a temperature gradient $G$, and it is pulled at a constant velocity $V_{p}$ towards the cold region of the furnace. For low pulling velocities, the interface remains stable and planar but, when $V_{p}$ exceeds a critical value [5,6], the solidliquid interface undergoes the Mullins-Sekerka morphological instability. The interface then develops fingerlike structures called cells and, for even higher velocities, dendritic structures with secondary and tertiary side arms. Further understating of the basic physical mechanisms governing microstructure formation during solidification are of major importance because they directly benefit to the improvement of materials processing [2,3].

The cellular or dendritic patterns may undergo secondary instabilities in domains of control parameters where nonsteady-state growth solutions become dynamically selected.

*Corresponding author: fatima.lisboa-mota@im2np.fr
Interface patterns may present a diversity of secondary instabilities, very frequently occurring in a narrow range of growth conditions [7-12]. One typical example of secondary instability, generic of spatially modulated interface patterns, is the oscillatory growth mode. In directional solidification, the period-doubling oscillatory mode $(2 \lambda-\mathrm{O})$ has been experimentally, theoretically, and numerically studied, in thin samples for both cellular [7,8,12-16] and two-phase eutectic interfaces [17-21]. Oscillatory modes have been predicted by numerical studies of 3D cellular growth first [22,23] before being recently experimentally observed [24-26].

In thin samples of transparent alloys, Georgelin and Pocheau [14] experimentally evidenced and characterized the oscillation of cellular patterns, that appeared to be a $2 \lambda-\mathrm{O}$ mode. Adjacent cells form pairs that oscillate in phase opposition with both tip position and cell width oscillation, nearly in phase quadrature. Recently, the origin and nature of oscillations was revisited by combining the former experiments of Georgelin and Pocheau [14] with 3D phase-field numerical simulations [16]. Phase-field simulations together with a solute balance model revealed the fundamental role of transverse solute fluxes between neighboring cells and provided a complete description of the physical mechanism of $2 \lambda-\mathrm{O}$ oscillations.

Due to gravity-induced convection, 3D oscillating modes were never directly observed in experiments until recently when experiments were performed under microgravity conditions in bulk (3D) samples of a succinonitrile-0.24 wt\% camphor alloy in the framework of joint project between the 
French Space Agency (CNES) and NASA. Experiments were performed using the Directional Solidification Insert (DSI) of the DEvice for the study of Critical LIquids and Crystallization (DECLIC) developed by CNES which is dedicated to in situ and real-time characterization of the dynamical selection of the solid-liquid interface morphology on bulk samples of transparent materials [27-30]. For a limited range of experimental control parameters, extended cellular patterns, that are globally disordered, form and display oscillations with periods of few tens of minutes. Oscillating cells are usually noncoherent due to array disorder. However, in regions displaying short-range spatial ordering, groups of cells can synchronize into oscillatory breathing modes. In case of a local hexagonal ordering, three sublattices beating with an approximately $\pm \frac{2 \pi}{3}$ phase shift with each other were observed; for a local square ordering, two sublattices were beating in phase opposition [24,25]. Dedicated large-scale phase-field simulations were performed that produced oscillating modes of comparable characteristics in terms of period as well as spatiotemporal coherence $[24,26]$. In simulations, long-range coherence of breathing modes could be achieved for a perfect spatial arrangement of cells imposed as initial condition, whereas, when realistic noisy initial conditions are used, a global disorder was observed that prevents oscillation coherence as in experiments. The conditions of appearance of oscillations were also specified and linked to the stable spacing range of a cellular array [26].

In the previous works [24-26], 3D oscillating experimental patterns were analyzed using the top-view images provided in DECLIC-DSI; in this observation mode, oscillation is revealed by the periodic variation of the apparent cell area which corresponds to the cell width oscillation seen from the top. The characterization of the cell shape is therefore only partial as tip shape and position are not measurable. As previously mentioned, all these parameters were measured for the case of thin samples observed from the side and were found to be affected by the oscillation [14]. To overcome this limitation and completely characterize the oscillating cell dynamics in the 3D extended patterns, we used the DECLIC-DSI MachZehnder interferometer which is installed along the axial direction of the crucible. This interferometer was specifically designed to capture the 3D geometry of the interface in real time. In the frame of the DECLIC project, interferometric data were previously used to quantitatively characterize the shape of dendrites grown by directional solidification and followed by in situ observation during their whole growth [31]. In this paper, the interferometric images of a reference experiment, whose control parameters lead to oscillating cellular patterns $\left(V_{p}=1 \mu \mathrm{m} / \mathrm{s}\right.$ and $\left.G=19 \mathrm{~K} / \mathrm{cm}\right)$, are analyzed to reconstruct the shape and position of each cell during its oscillation cycle and rebuild the oscillation cycle of the main cell shape parameters, tip position, radius, and velocity. Dedicated phase-field simulations are used to better understand the driving mechanisms of cellular oscillation. The results shed light on the mechanisms of oscillation which involves vertical and horizontal solute fluxes.

In Sec. II the experimental device will be first briefly described as well as the method of interferometric data treatment used to exploit the information encoded in the interferometric fringes. It produces detailed quantitative descriptions of the interface shapes and their time evolution. Tip velocity variations can be evidenced with resolution of a few nanometers per second. Section III covers the statistical characteristics and mechanism of oscillation of individual cells, including a detailed description of the quantities and phase relationships involved in an oscillating cell, the supporting phase-field simulations and a proposed driving mechanism of cellular oscillation. Conclusions will be summarized in Sec. IV.

\section{EXPERIMENTAL}

\section{A. The directional solidification device DECLIC-DSI}

The DSI insert of the DECLIC facility includes a Bridgman-type furnace and the experimental cartridge; more complete descriptions of DECLIC and its inserts can be found elsewhere $[29,30]$. The Bridgman furnace is composed of hot and cold zones located at the top and bottom of the adiabatic zone, respectively, so that a temperature gradient $G$ can be generated (between 10 and $30 \mathrm{~K} / \mathrm{cm}$ ). Solidification is performed by pulling the experimental cartridge containing the alloy from the hot zone towards the cold zone at a constant pulling rate $V_{p}$ (between 0.1 and $30 \mu \mathrm{m} / \mathrm{s}$ ). The experimental cartridge includes the quartz crucible and a system of volume compensation made of stainless steel that is mandatory to accommodate the specimen volume variations associated to phase changes. The cylindrical crucible has an inner diameter of $1 \mathrm{~cm}$ and a length that enables about $10 \mathrm{~cm}$ of solidification, thus allowing the study of the whole development of extended 3D patterns from their initial stages till permanent regime of growth morphology. The crucible is equipped with a flat glass window at the bottom and with an immersed lens in the melt at the top. The main observation mode takes advantage of the complete axial transparency of the cartridge provided by these last two elements: the light coming from LEDs passes through the cartridge from the bottom to the top, therefore crossing the interface, the image of which is formed on a CCD camera (top-view image); this observation mode provides top-view images of the microstructure and is used to study array dynamics and cell characteristics. On the same cartridge axis, a Mach-Zehnder interferometer is also set using a He-Ne laser $(\lambda=632.8 \mathrm{~nm})$. The basic principles of the analyses of the interferometric images can be found in Refs. [31,32] but the ultimate method that we currently use will be presented in Sec. II B. In the transverse mode, the light coming from two LEDs crosses the sample from one side to the other, which provides a real-time control of interface position and curvature (side-view image). Due to the cylindrical shape of the crucible, this observation mode has a quite low resolution and cannot be used for the detailed analysis of cell shape. In this study, only the images obtained by the direct and interferometric axial observations are analyzed. It is worth noticing that since those two diagnostics share the same optical path, they cannot be active simultaneously.

The organic transparent alloy Succinonitrile (SCN) 0.24 wt $\%$ camphor is used (Table I). SCN purified by NASA by both distillation and zone melting was used to fill the crucible. All procedures for sample preparation were carefully realized under vacuum to avoid humidity contamination. A single crystalline solid seed with a direction $\langle 100\rangle$ parallel to 
TABLE I. Properties of succinonitrile and succinonitrilecamphor alloy [33-39].

\begin{tabular}{lccc}
\hline \hline Physical parameter & Symbol & Magnitude & Unit \\
\hline \multicolumn{4}{c}{ Succinonitrile } \\
& $T_{m}$ & 331.24 & $\mathrm{~K}$ \\
Melting temperature & $M$ & 80.09 & $\mathrm{~g} / \mathrm{mol}$ \\
Molar mass & $\Delta H$ & 3.713 & $\mathrm{~kJ} / \mathrm{mol}$ \\
Latent heat of fusion & $\rho_{L}$ & 970 & $\mathrm{~kg} / \mathrm{m}^{3}$ \\
Liquid density & $\rho_{S}$ & 1016 & $\mathrm{~kg} / \mathrm{m}^{3}$ \\
Solid density & $\lambda_{L}$ & 0.223 & $\mathrm{~J} /(\mathrm{ms} \mathrm{K})$ \\
Liquid thermal conductivity & $\lambda_{S}$ & 0.224 & $\mathrm{~J} /(\mathrm{ms} \mathrm{K})$ \\
Solid thermal conductivity & $\beta_{T}$ & $7.85 \times 10^{-4}$ & $\mathrm{~K}-1$ \\
Thermal expansion coefficient & $\gamma$ & $8.95 \times 10^{-3}$ & $\mathrm{~J} / \mathrm{m}^{2}$ \\
Interface energy & $v$ & 2.6 & $\mathrm{~mm}{ }^{2} / \mathrm{s}$ \\
Kinetic viscosity & Succinonitrile-camphor & \\
\hline Solute (camphor) concentration & $C_{0}$ & 0.24 & $\mathrm{wt} \%$ \\
Liquidus slope & $m$ & -1.365 & $\mathrm{~K} / \mathrm{wt} \%$ \\
Solute diffusion coefficient (liq.) & $D_{L}$ & 270 & $\mu \mathrm{m}^{2} / \mathrm{s}$ \\
Thermal diffusion coefficient & $D_{t h}$ & $1.15 \times 10^{5}$ & $\mu \mathrm{m}^{2} / \mathrm{s}$ \\
Solute partition coefficient & $k$ & 0.07 & - \\
Gibbs-Thomson coefficient & $\Gamma$ & 0.06478 & $\mathrm{~K} \mu \mathrm{m}$ \\
Anisotropy strength & $\epsilon_{4}$ & 0.011 & - \\
\hline \hline
\end{tabular}

the pulling axial direction was prepared on ground and kept during all the experimental campaign. Further details about the experimental procedure can be found in previous works $[28,40]$.

No direct in situ measurement of the thermal gradient is available in the DSI as no thermocouple is inserted inside the sample to avoid thermal perturbations. A complete discussion of the thermal gradient determination can be found in Ref. [41]: two different thermal gradients were estimated by thermal numerical simulation [42] at $G_{1}=19 \mathrm{~K} / \mathrm{cm}$ and $G_{2}=12 \mathrm{~K} / \mathrm{cm}$. A range of pulling velocities from 0.25 to 30 $\mu \mathrm{m} / \mathrm{s}$ was studied which covers the whole range of possible unstable microstructures.

The oscillation mode was observed in a narrow range of pulling rates: from 0.5 to $1.5 \mu \mathrm{m} / \mathrm{s}$ for a thermal gradient of $19 \mathrm{~K} / \mathrm{cm}$, and at $0.5 \mu \mathrm{m} / \mathrm{s}$ for a gradient of $12 \mathrm{~K} / \mathrm{cm}$. The most striking oscillatory experiment, which was the basis of our previous analyses, was obtained for $V_{p}=1 \mu \mathrm{m} / \mathrm{s}$ and $G=19 \mathrm{~K} / \mathrm{cm}[24,25]$. These last conditions are then chosen to perform the experiment with sequences of interferometric observation followed by white light acquisitions of the same duration (approximately $45 \mathrm{~min}$ ).

\section{B. Interferometry in the context of cellular interface characterization}

Interferometric images are used to determine the interface shape and growth velocity. Interference fringes appear at the recombination of the laser beam passing through the crucible with a reference beam passing outside. These fringes can be considered as level curves of the solid-liquid interface. The spatial variation of the interferogram contains the information related to the 3D shape of the cell tips, whereas its temporal evolution is required to follow the height of an in-
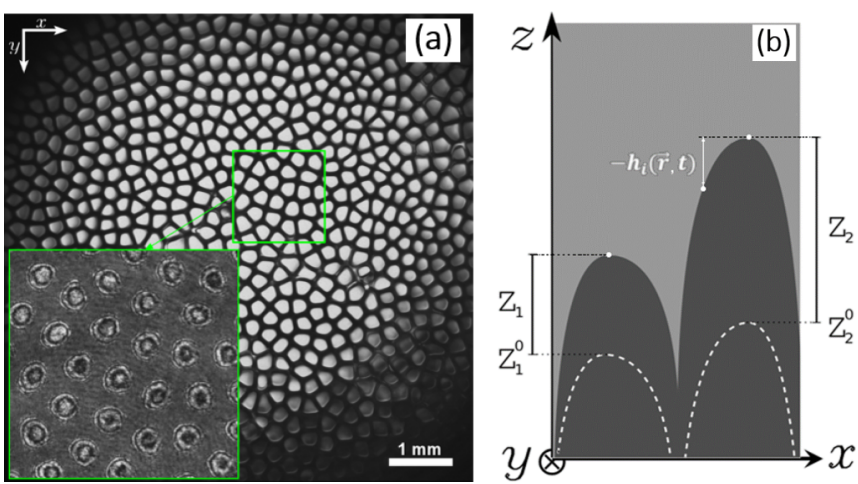

FIG. 1. (a) Global view of the solid-liquid interface obtained by direct observation $\left(V_{p}=1 \mu \mathrm{m} / \mathrm{s}, G=19 \mathrm{~K} / \mathrm{cm}, t=15.6 h\right)$. The green square is zoomed to show the corresponding interferometric observation. (b) Frames of reference used for interferometric reconstruction. The instantaneous position $Z_{i}$ of each cell is taken relative to its initial position $Z_{i}^{0}$ defined at $t=0$. The shape of a cell is described by the height of the interface $h_{i}(\vec{r}, t)$, which is a function of $\vec{r}$, a $2 \mathrm{D}$ vector with origin fixed at the cell tip position.

terface point with time [31]. A snapshot of a cellular interface obtained by direct and interferometric observations is given in Fig. 1(a). In the interferometric view, each group of rings corresponds to a cell, separated from its neighbors by a region where beam coherence is lost. Therefore, the interferogram conveys information only near the cell tips; as there are no fringes in the grooves between cells, it is not possible to directly relate the tip heights of different cells. Temporally, we can obtain only the variation of an interface point height relative to its initial height at the start of interferometric acquisition but not its absolute height.

We define $Z$ as the pulling and optical axis. The simplest representation of a cellular interface is a height map $z_{\phi}(\vec{r}, t)$ with $\vec{r}$ the location of an interface point in the $(x, y)$ plane normal to $Z$ [Fig. 1(b)] and the subscript $\phi$ stands for the tip phase shift, which will be detailed in the next subsection. As there is no continuity of fringes from one cell to its neighbors, it is convenient to separate $z_{\phi}(\vec{r}, t)$ into the tip-position component $Z_{i}(t)$ and the shape component $h_{i}(\vec{r}, t)$ :

$$
z_{\phi}(\vec{r}, t)=Z_{i}(t)+h_{i}(\vec{r}, t) .
$$

The frames of reference $\vec{r}$ and $h$ are centered on the tip position of the $i$ th cell [Fig. 1(b)].

We can detail the variation of the tip height:

$$
Z_{i}(t)=Z_{i}^{0}+V_{p} t+\delta Z_{i}(t),
$$

where $Z_{i}^{0}$ is the initial height of the ith's cell tip at the start of the sequence (it depends exclusively on $i$ ), $V_{p} t$ is the average front elevation during growth and $\delta Z_{i}(t)$ is the possible deviation of a cell tip from this average, resulting for example from velocity oscillations (it depends on $i$ and $t$ ). The position of the interface $z_{\phi}(\vec{r}, t)$ can then be separated into an absolute front position component $Z_{\text {ifront }}(t)=Z_{i}^{0}+V_{p} t$, a relative cell tip position component $\delta Z_{i}(t)$ and a shape component $h_{i}(\vec{r}, t)$ :

$$
z_{\phi}(\vec{r}, t)=Z_{\text {ifront }}(t)+\delta Z_{i}(t)+h_{i}(\vec{r}, t) .
$$

The main objectives are then to calculate $h_{i}(\vec{r}, t)$ to access the interface shape, and to calculate $\delta Z_{i}(t)$ and its time 

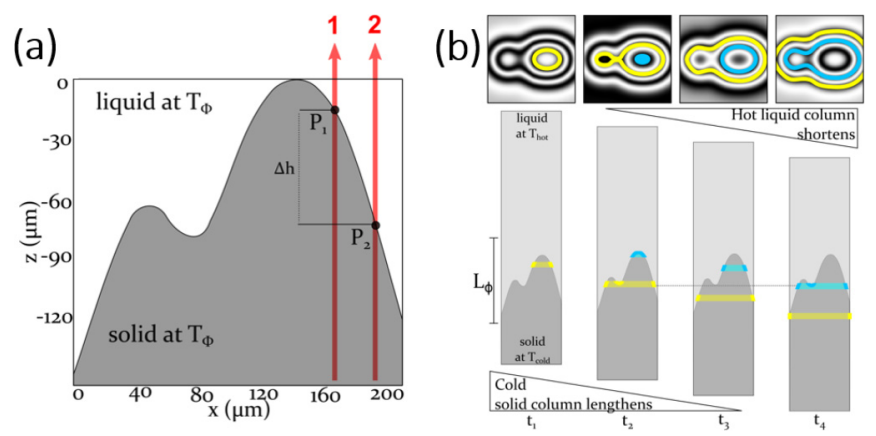

FIG. 2. (a) Side cut of the solid-liquid interface. Two adjacent rays 1 and 2 have similar paths save for the short distance $\Delta h$ where $P_{1}$ travels through a solid, and $P_{2}$ travels through a liquid. Their difference in phase can then be attributed solely to the shape of the interface. (b) Time evolution: as pulling rate is applied, the entire interface advances, and the fringes slide towards lower parts of the interface. The interface shape is considered constant within short durations, and the temporal change in $L$ is attributed solely to changes in length of the solid and liquid columns above and below the interface.

derivative to characterize the oscillation of the cell tip in terms of height and velocity. Each cell is treated independently. The starting point is the interferogram and its spatiotemporal intensity variations.

\section{From the interferogram to the cell tip characterization}

For a point of location $z_{\phi}(\vec{r}, t)$ attached to a given cell, the corresponding intensity $I(\vec{r}, t)$ recorded at instant $t$ on the CCD camera results from the difference of optical paths $L(\vec{r}, t)$ between the ray crossing the interface at $\vec{r}$, and its corresponding reference ray:

$$
I(\vec{r}, t)=2 I_{0}\{1+\cos [\varphi(\vec{r}, t)]\} \text { with } \varphi(\vec{r}, t)=2 \pi \frac{L(\vec{r}, t)}{\lambda} .
$$

$\varphi(\vec{r}, t)$ is simply the optical path phase difference at a given point $\vec{r}$ and time $t$; it can be rewritten in analogy to $z_{\phi}$ :

$$
\varphi(\vec{r}, t)=\phi_{i}(t)+\theta_{i}(\vec{r}, t),
$$

where $\phi_{i}(t)$ is the tip phase shift taken at the tip position due to $Z_{i}(t)$, and $\theta_{i}(\vec{r}, t)$ is the component of the phase shift due to the shape component $h_{i}(\vec{r}, t)$. These phase differences are linked to the optical paths:

$$
L_{S i}(\vec{r}, t)=\frac{\theta_{i}(\vec{r}, t) \lambda}{2 \pi} \text { and } L_{T i}(t)=\frac{\phi_{i}(t) \lambda}{2 \pi},
$$

where $L_{S i}$ corresponds to the part of $L$ due to shape [i.e., related to $\left.h_{i}(\vec{r}, t)\right]$, and $L_{T i}$ corresponds to the part of $L$ due to the tip height [i.e., related to $\left.Z_{i}(t)\right]$.

During a solidification experiment, the main variations of optical paths are attributed to variations of the lengths of the two different phases (solid and liquid). The optical paths of parallel rays crossing the interface at different locations $P_{1}$ and $P_{2}$ at the same time $t$ [Fig. 2(a)] reveal the interface shape. If $P_{1}$ and $P_{2}$ lie on two neighboring black fringes [blue and yellow lines in Fig. 2(b)], their $L$ differs by a laser wavelength $\lambda$. Similarly in time, if a pixel is at its darkest at a given time, the next time it will be at its darkest again is when its $L$ is one laser wavelength $\lambda$ longer.

The optical path $L$ depends both on the position of the solid-liquid interface $z_{\phi}(\vec{r}, t)$, and of the entire temperature field of the sample $T(\vec{r}, z, t)$, because of the dependence of the optical index on the temperature. In DECLIC-DSI, there is no access to the temperature field, so a simplified model is used as shown in Fig. 2. Homogeneous temperature $T_{\phi}$ along the interface and its close vicinity is assumed. Far from the interface, the liquid is at temperature $T_{\text {hot }}$, and the solid is at $T_{\text {cold }}$. The shape component is obtained by comparing the optical paths of two rays crossing the interface at different locations, namely, at $\vec{r}$ and at the tip $(\vec{r}=\overrightarrow{0})$. The corresponding paths differ only close to the interface over a length $h(\vec{r})$ :

$$
\begin{aligned}
L_{S i}(\vec{r}, t) & =L(\vec{r}, t)-L(\overrightarrow{0}, t) \\
& =-h(\vec{r}) n_{\phi L}+h(\vec{r}) n_{\phi S} \\
\Leftrightarrow h(\vec{r}) & =\frac{L_{S i}(\vec{r}, t)}{\Delta n_{\phi}},
\end{aligned}
$$

where $n_{\phi L}$ and $n_{\phi S}$ are the refractive indices at the interface temperature on the liquid and the solid side, respectively, and $\Delta n_{\phi}=n_{\phi S}-n_{\phi L}$. The time component is obtained by following the $L$ variation at one location. Due to the motion of the crucible, a length $V_{p} \Delta t$ of cold solid replaces the same length of hot liquid during a time $\Delta t$. If the local tip velocity varies about the pulling velocity (in case of oscillation for example), the tip location is shifted by $\delta Z_{i}(t)$ ahead or below the average interface location (steady-state growth). If $\delta Z_{i}(t)>0$, a corresponding length of solid at temperature $T_{\phi}$ has replaced the liquid during $\Delta t$ :

$$
L_{T i}(t)=L(\overrightarrow{0}, t+\Delta t)-L(\overrightarrow{0}, t)=V_{p} \Delta t \Delta n_{S L}+\delta Z_{i}(t) \Delta n_{\phi},
$$

where $\Delta n_{S L}$ is the difference of refractive indices between the cold solid and hot liquid $\left(\Delta n_{S L}=n_{S T_{\text {cold }}}-n_{L T_{\text {hot }}}\right)$, and in steady state $\delta Z_{i}(t)=0$.

Several measurements were performed to determine the variation of the refractive index of pure liquid $\mathrm{SCN}$ as a function of temperature (Inatomi et al. [43] and unpublished data by Trivedi et al.). The solid phase was explored less, and the only extensive characterization of $n(T)$ was done by MacFarlane et al. [44]. From the literature data, $\Delta n_{S L}=0.034$ and $\Delta n_{\phi}=0.019$. These values are critical to the characterization of the interface, so they have been reassessed in situ during the experimental campaign in microgravity [45]. The value of $\Delta n_{\phi}$ was refined to 0.013 by analyzing the interferogram of a macroscopically curved interface, whose curvature was observed by transverse observation. The value of $\Delta n_{S L}$ was refined to 0.0348 by counting precisely the number of fringes that traversed the middle of the interface during a long experiment of precisely known pulling length. These latter values are used for analysis.

Based on Eqs. (4)-(9), we see that to reconstruct the interface shape and to get the local tip velocities, the interferograms need to be analyzed to extract first the phase shift and then to deduce the different components of optical paths $L_{S i}$ and $L_{T i}$. The problem is then to extract an adequate phase shifting that monotonously describes the tip profile, in spite of the periodic variation of the intensity, and in spite 
of unavoidable noise in intensity variations of the interferograms. To solve this problem, the phase-shifting algorithm proposed by Wang and Han [46] was implemented in an inhouse procedure written in Python with extensive use of the open-source scientific libraries NumPy and SciPy. At instant $t$, a small subset of consecutive interferograms, of sufficiently short duration to consider that the cell shape is not changing, is used to calculate the phase-map image $\theta_{i}(\vec{r}, t)$ that reflects the morphology; successive subsets of images superpose to ensure the continuity of the results. The successive subsets are used to determine $\phi_{i}(t)$.

The duration of an interferometry sequence is $45 \mathrm{~min}$, which amounts to $\approx 800$ images. The treatment was applied to an interface area of $5.0 \times 4.3 \mathrm{~mm}^{2}$ enclosing $M=337$ cells. To analyze the oscillating cells, gliding subsets of 30 images, with a shift of three images between successive subsets, are used. For each cell, the result of the treatment is a curve corresponding to $Z_{\text {ifront }}(t)+\delta Z_{i}(t)$ and a sequence of cell shapes $h_{i}(\vec{r}, t)$ at different times. Due to the lack of an absolute reference, inherent to interferometry, the origin of $Z$ is taken to be 0 , meaning that $Z_{i}^{0}=0$ and $Z_{\text {ifront }}(t)=V_{p} t$. This procedure was repeated for each observed cell, the $Z$ position of the cell tips was averaged, and the average was taken as the front position $Z_{\text {front }}(t)=\frac{1}{M} \sum_{i=1}^{M} z_{\phi, i}(\overrightarrow{0}, t)=V_{p} t=Z_{\text {ifront }}(t)$. Subtracting this average component from $z_{\phi, i}(\vec{r}, t)$, the two characteristics affected by oscillation, $\delta Z_{i}(t)$ and $h_{i}(\vec{r}, t)$, can be obtained from Eq. (3).

To calculate the growth velocity deviation $\delta V_{i}(t)\left[\frac{d \delta Z_{i}(t)}{d t}\right]$ from the raw measured data, $\delta Z_{i}(t)$ is smoothed by applying a Savitzky-Golay filter (windowed linear least squares fit) with a window of $3 \mathrm{~min}$, before being differentiated with respect to time. A filter window of $3 \mathrm{~min}$ was chosen by trial and error to reduce noise without significantly altering the shape of the curve.

As mentioned above, only the regions near the tips are accessible through interferometry. The lowest observable point of $h_{i}(\vec{r}, t)$ is typically $300 \mu \mathrm{m}$ below the cell tip. These regions have a mean diameter of $86 \mu \mathrm{m}$, in contrast to $154 \mu \mathrm{m}$ considering the apparent cell areas during direct observation. The measurement of tip radius is chosen to quantitatively characterize the cell shape and its evolution. An elliptical paraboloid is fitted to the reconstructed depth map $h_{i}(\vec{r}, t)$ which allows estimating the mean radius of curvature $\rho_{i}(t)$ for each cell. Considering the mean radius of curvature or Gaussian radius of curvature leads to very similar results, so it was decided to consider only the mean radius.

\section{RESULTS}

Before turning to the cell oscillation dynamics, it is important to briefly describe the main characteristics of the extended 2D cellular pattern. The in situ and real-time observation of the solidification allows one to follow the primary spacing evolution as function of time. Primary spacing is one of the main characteristics describing the interface morphology and is defined as the average center-to-center distance between a cell and its first neighbors. The average primary spacing increases until it reaches a steady-state value. If an average is made throughout the whole interface, the primary spacing at steady state is equal to $288 \pm 42 \mu \mathrm{m}$ which compares
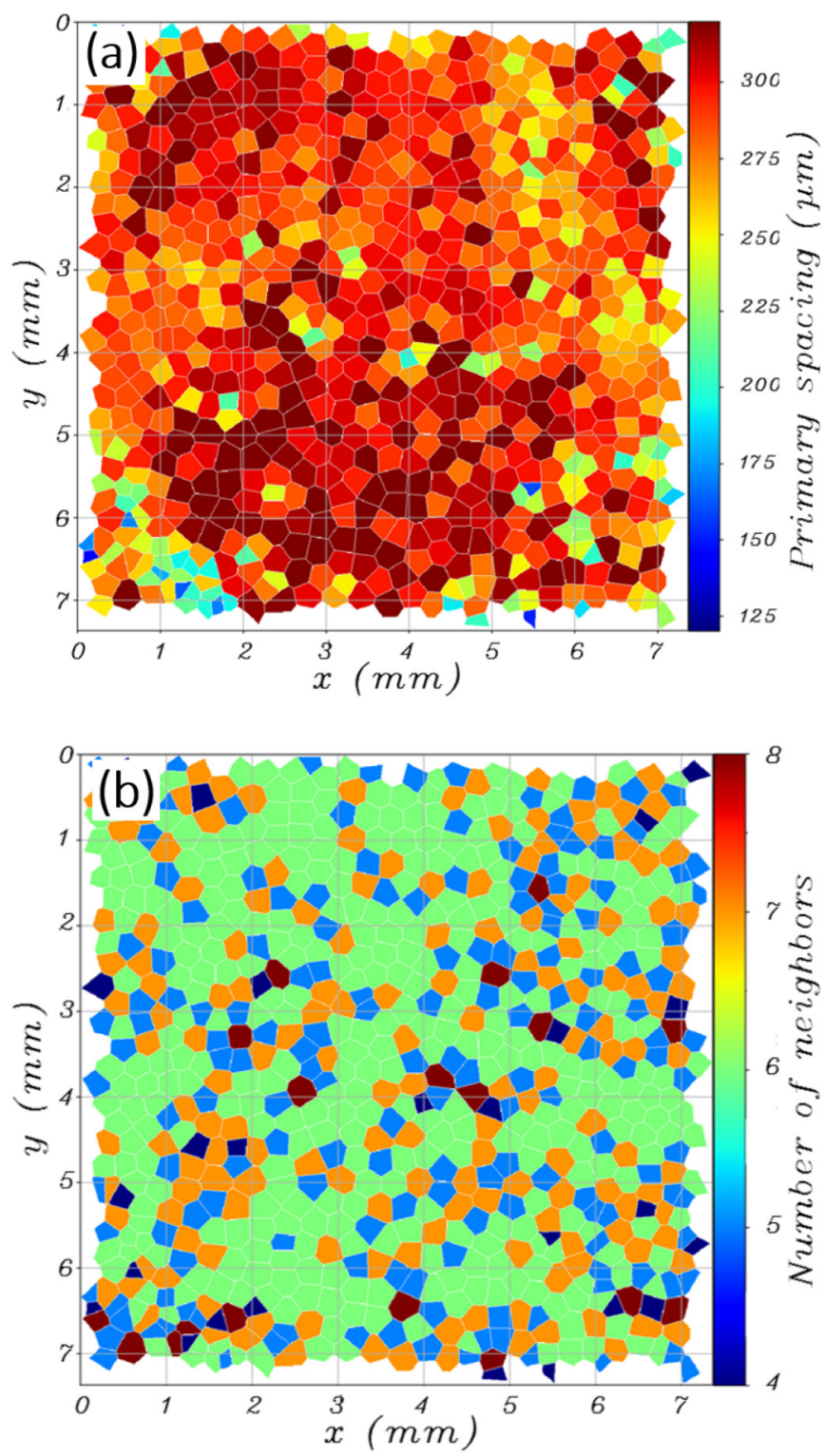

FIG. 3. Maps of primary spacing (a) and nearest neighbors (b) at steady state $\left(V_{p}=1 \mu \mathrm{m} / \mathrm{s}, G=19 \mathrm{~K} / \mathrm{cm}, t=15.6 h\right)$.

well with the previously analyzed experiment (without interferometry) [25]. The primary spacing map at the end of solidification is given in Fig. 3(a): the primary spacing is homogeneously dispersed and does not depend on the cell's placement on the interface. The number of nearest neighbors is also determined which gives information on the quantity of topological defects in the pattern: a perfect hexagonal tiling would correspond to six nearest neighbors for each cell. Figure 3(b) shows the map of nearest neighbors at steady state: there is a high number of topological defects that are mainly instances of five and seven nearest neighbors. Similar to our previous work [24-26], the experiments show a complex interface dynamics, with a constantly evolving spatial organization of cells, nourished by regular tip-splitting and elimination events. The pattern does not display any longrange order. 


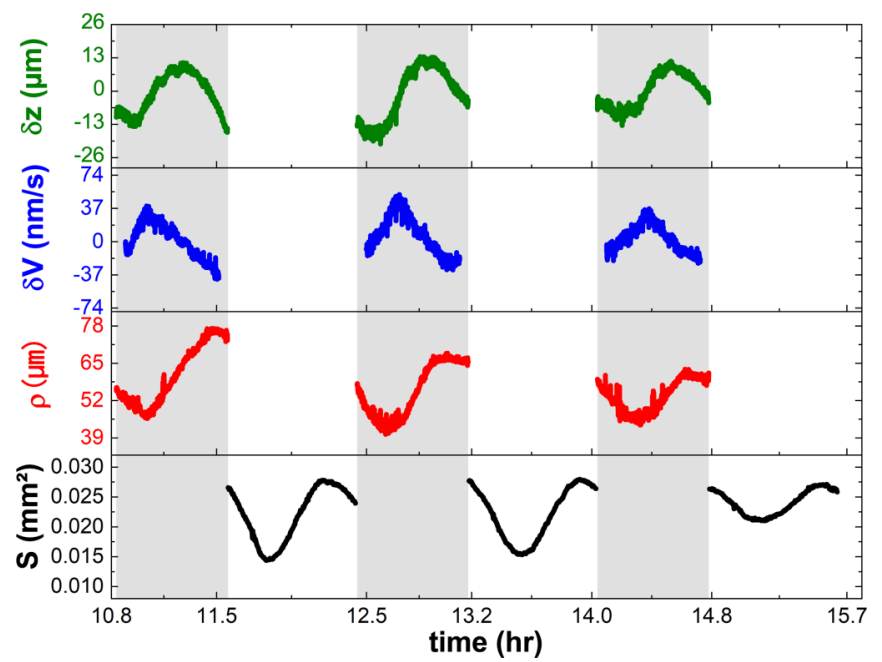

FIG. 4. Example of the data obtained for a single cell during three interferometric (shaded region) and three direct observation sequences. $\delta Z, \delta V, \rho$, and $S$ have oscillation amplitudes of $11 \mu \mathrm{m}$, $26 \mathrm{~nm} / \mathrm{s}, 12 \mu \mathrm{m}$, and $0.005 \mathrm{~mm}^{2}$, respectively $\left(V_{p}=1 \mu \mathrm{m} / \mathrm{s}, G=\right.$ $19 \mathrm{~K} / \mathrm{cm})$.

\section{A. Oscillation of cell tip parameters}

Each interferometric or white light acquisition sequence represents approximately one period of the oscillations. The white light acquisitions yield the evolution of the cell visible surface $S(t)$, and the interferometric acquisitions yield the radius of curvature evolution $\rho_{i}(t)$, and cell position deviation $\delta Z_{i}(t)$. Figure 4 shows an example of the data extracted from the sequences of direct and interferometric observations of one cell: pseudo-sinusoidal periodic oscillations are evidenced. A movie of the evolution of one cell throughout the sequences is given in the Supplemental Material Ref. [47] to visually check that the same cell has been followed throughout. The data are then fitted with a sine function $y(t)=$ $A \sin \left(\frac{2 \pi}{T} t+\phi\right)+B$, where $A$ is the amplitude, $T$ is the period, $\phi$ is the phase and $B$ the offset. The physical quantities $\delta Z_{i}(t)$, $\delta V_{i}(t), \rho_{i}(t)$, and $S_{i}(t)$ are fitted simultaneously, using the same period throughout the fit, but different amplitudes, phase shifts, and offsets.

Statistical analyses conducted on 336 cells enable us to evaluate the amplitudes of oscillations of all these data, which appear to vary widely from one cell to the other. Some cells do not oscillate, or the amplitude of their oscillation is too small to be measured, other cells may present a phase shift in oscillation so that the fit is not accurate. The 104 cells with the best sinusoidal fit were used below. Sinusoidal fits of $\delta Z$ and $S$ exhibit a similar mean period of $52 \pm 6 \mathrm{~min}$. These measurements are close albeit slightly higher than the average value obtained for the other solidification experiment at $V_{p}=$ $1 \mu \mathrm{m} / \mathrm{s}$ that was observed in white light (48 min) [24-26]. Typical amplitudes of oscillation of tip position, growth velocity, and tip radius are, respectively, of $12 \mu \mathrm{m}(\approx 1 \%$ of the thermal length), $25 \mathrm{~nm} / \mathrm{s}$ ( $\approx 3 \%$ of the pulling velocity), and $9 \mu \mathrm{m}(\approx 17 \%$ of the average tip radius), even if quite large dispersions are observed. The order of magnitude of the oscillation amplitudes is in good agreement with the one measured
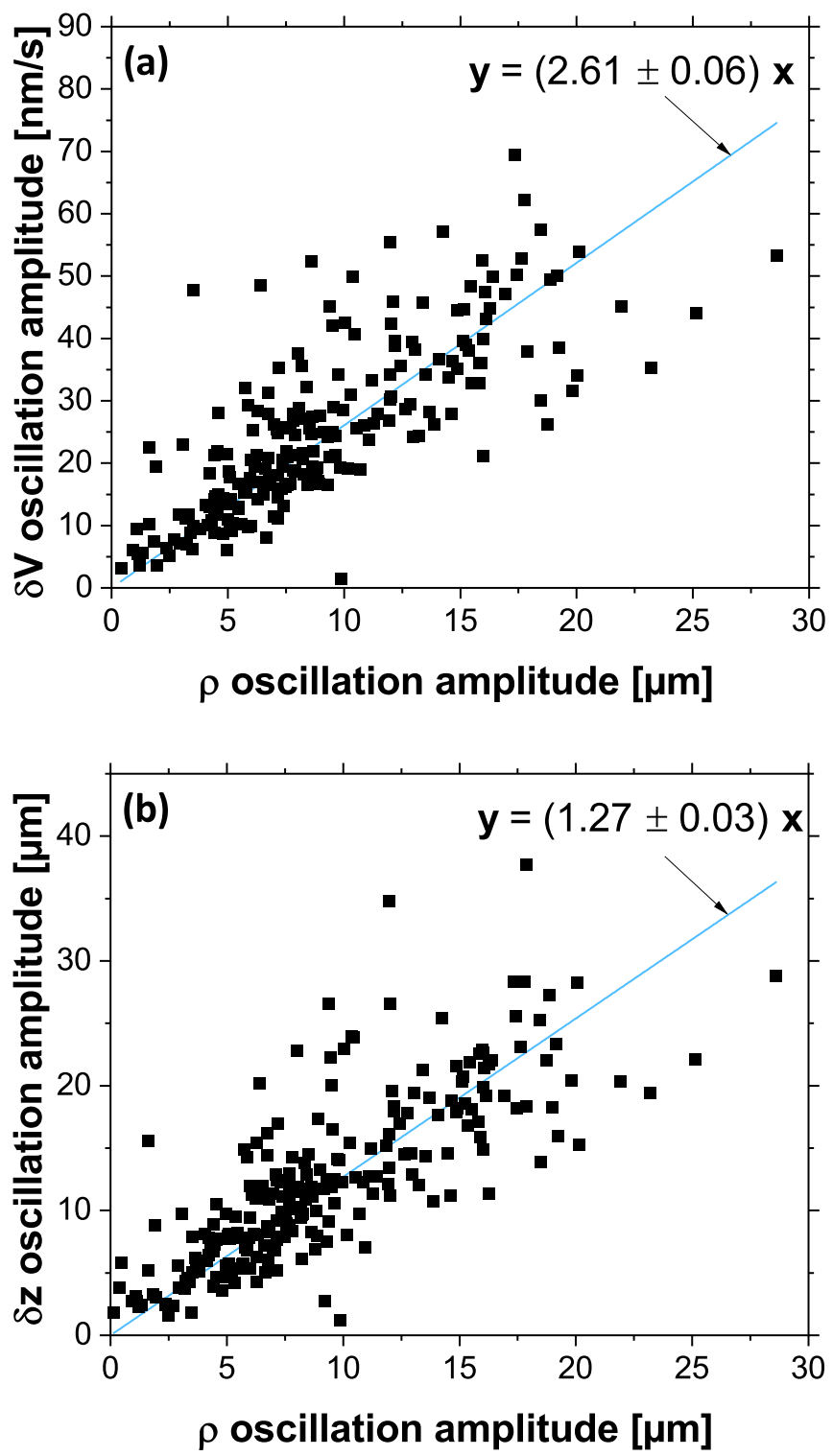

FIG. 5. Amplitudes of oscillation of the data extracted from interferometric measurements. Amplitudes for growth velocity (a) and tip position (b) are represented as a function of the tip radius oscillation amplitudes, to reveal their correlations $\left(V_{p}=1 \mu \mathrm{m} / \mathrm{s}, G=\right.$ $19 \mathrm{~K} / \mathrm{cm})$.

in $2 \lambda-O$ modes in thin samples [14]. As expected, amplitudes of oscillation of these different characteristics extracted from interferometry measurements show marked correlations as illustrated in Fig. 5: $A_{\delta V}[\mathrm{~nm} / \mathrm{s}]=(2.61 \pm 0.06) A_{\rho}[\mu \mathrm{m}]$ and $A_{\delta z}[\mu \mathrm{m}]=(1.27 \pm 0.03) A_{\rho}[\mu \mathrm{m}]$.

The next step is to analyze the phase relations between the different oscillating variables. To obtain phase relationships between $S(t)$, extracted from direct observation, and $\delta Z$ and $\rho$, extracted from interferometric measurements that are not simultaneous, the curves are individually fitted to sinusoids, and the phases of the fits are compared. The histogram of Fig. 6(a) depicts the phase difference between $\rho(t)$ and $S(t)$, whereas Fig. 6(b) points out the correlation of their amplitudes $\left[A_{S}\left[\mathrm{~mm}^{2}\right]=(9.861 \pm 0.01) \times 10^{-3} A_{\rho}[\mu \mathrm{m}]\right]$. It appears that the radii of curvature and the apparent cell areas are generally 

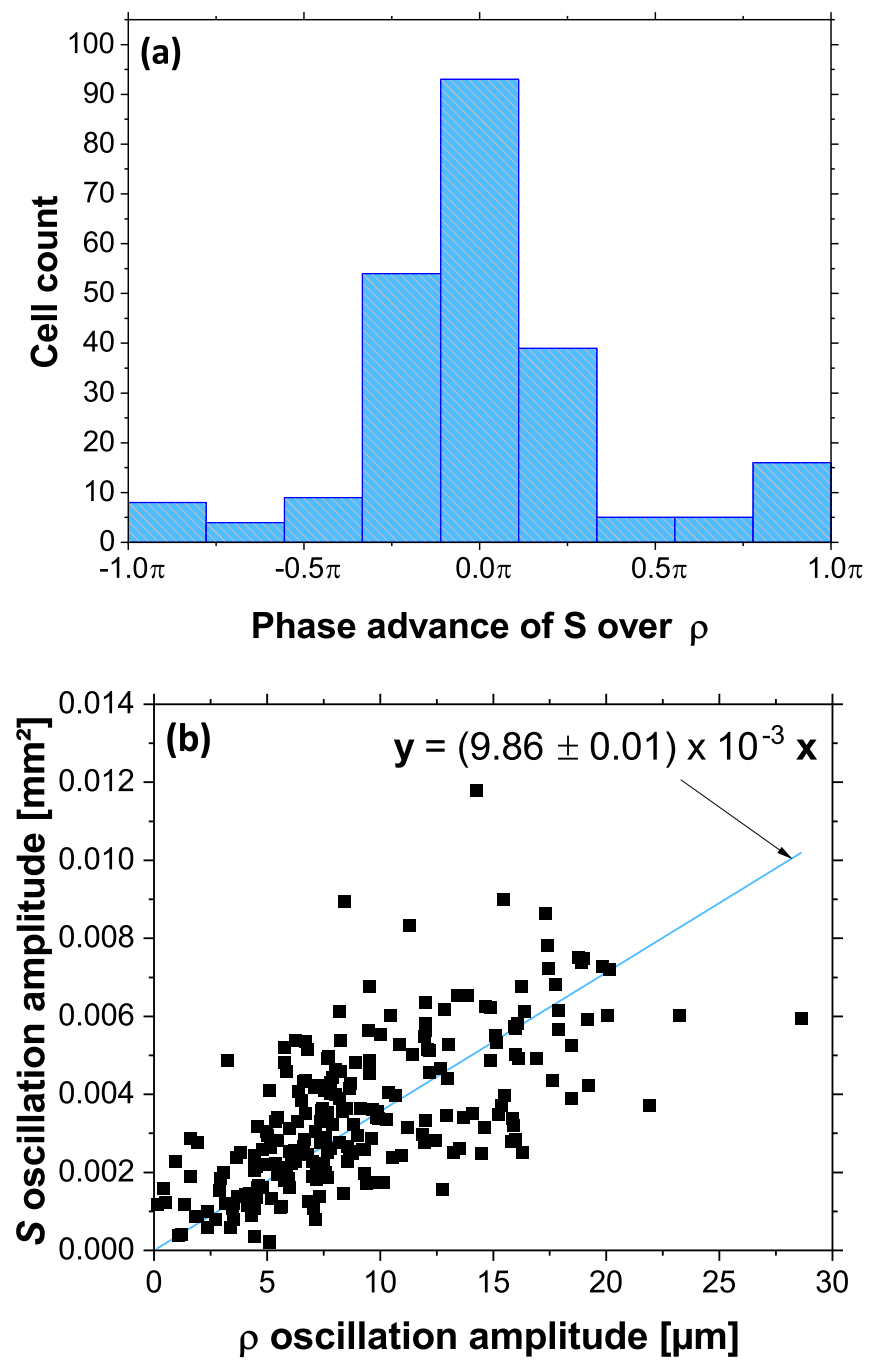

FIG. 6. Comparison of cell area, $S(t)$, and tip radius, $\rho(t)$, oscillations: (a) phase difference; (b) correlation of oscillation amplitudes $\left(V_{p}=1 \mu \mathrm{m} / \mathrm{s}, G=19 \mathrm{~K} / \mathrm{cm}\right)$. in phase and well correlated, albeit with a large dispersion. Regarding the fact that the size of the apparent surface area is limited mainly by the deviation of the light due to high slope towards the grooves, it can be considered that $S(t)$ and $\rho(t)$ roughly describe the same phenomenon: an increase of the tip radius corresponds to a larger and flatter tip therefore associated to an increase of $S(t)$. The large dispersion may be attributed to the fit quality as the data are not simultaneously acquired and are not perfectly sinusoidal. In the following, it will then be considered that $S(t)$ and $\rho(t)$ both describe the lateral stretching or shrinking of cells during oscillation and the focus will be on phase relations between $\rho, \delta Z$, and $\delta V$, which are obtained from the sine fits.

As illustrated in Fig. 4, growth velocity is in phase advance compared to tip position oscillation, which is itself in phase advance compared to tip radius (and apparent cell surface) oscillation. If the direct measurements of $\delta Z_{i}(t)$ and $\delta V_{i}(t)$ are compared for each one of the cells $i$, the average difference between $\delta Z$ and $\delta V$ is $\approx 0.52 \pi$, as expected from a pseudo-sinusoid and its derivative. As illustrated in Fig. 7, the

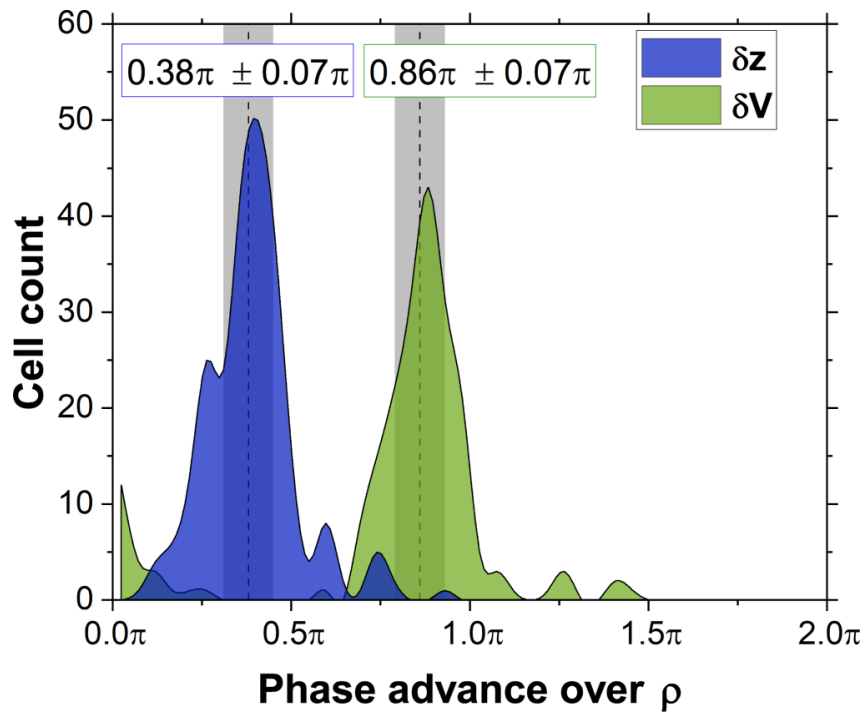

FIG. 7. Histograms of phase differences $\rho-\delta Z$ (dark blue) and $\rho-\delta V$ (light green). Only cells with the best sinusoidal fits are considered $(\approx 104$ cells). The gray intervals in each peak contain $\approx 60 \%$ of the cells.

phase shift between $\rho$ and $\delta V$ is $(0.86 \pm 0.07) \pi$; it is $(0.38 \pm$ $0.07) \pi$ between $\rho$ and $\delta Z$ (the defined intervals gather about $60 \%$ of the cells). Regarding these data, the difference between those phase shifts is equivalent to the phase difference between $\delta Z$ and $\delta V$ : slightly reduced to $\approx 0.48 \pi$, but in good agreement with the direct measurements. Figure 8 (a) shows an idealized plot of these curves based on the mean phase values: it can be seen as the evolution of the main characteristics for a typical oscillating cell.

The variations of cell tip surface and tip undercooling during oscillation predicted by previous 3D phase-field calculations for in-phase oscillation (Fig. 11 of Ref. [26]) can be compared to these values considering that the cell tip surface is in phase with the tip radius and that there is phase opposition between tip position and undercooling. With these assumptions, the phase-field results display a phase difference of $0.36 \pi$ between $\rho$ and $\delta Z$, consistent with our measurements. Recent phase-field simulations [16] applied to the experiments in thin samples of Georgelin and Pocheau [14] report phase shifts comparable with our values: $0.75 \pi$ between $\rho$ and $\delta V$, and $0.25 \pi$ between $\rho$ and $\delta Z$.

New 3D phase-field simulations have been performed in the frame of the analysis of this specific study and compared to experimental results. We will now refer to those simulations when comparing to our experiments. The phase-field model used is based on the thin interface phase-field model extended to the case of a binary dilute alloy [33], and is exactly the same as in Ghmadh et al. [16]. Further details of simulation procedures can be found in Ref. [26]. The model is adapted to the present situation by using the physical parameters for the SCN-camphor alloy given in Table I. Simulations are performed for $V_{p}=1 \mu \mathrm{m} / \mathrm{s}$ and $G=24 \mathrm{~K} / \mathrm{cm}$. As pointed out in our previous work, the thermal gradient used in phase field simulations is taken slightly higher than the experimental value to find stable oscillating patterns [26]. Coherent oscillations of three groups of cells in a hexagonal array are obtained 

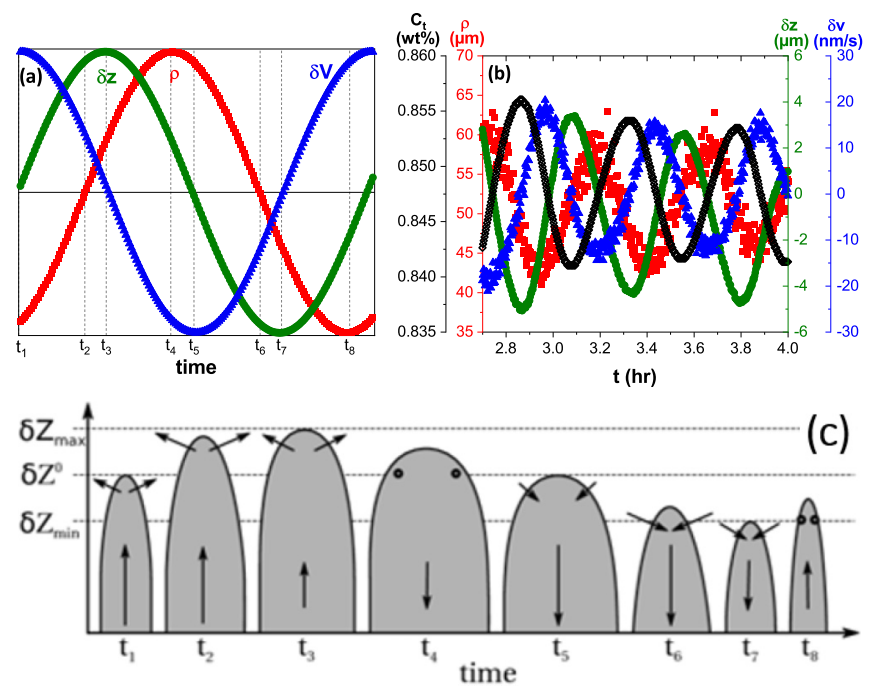

FIG. 8. (a) Scheme of the evolution of a cell shape during an oscillation period. Simplified representation of the $\delta V, \delta Z$, and $\rho$ curves of a cell, shown as sinusoids of normalized amplitude (markers blue $\boldsymbol{\Delta}$, green , and red $\boldsymbol{\square}$, respectively). These curves are generated from the mean of the differences of the studied cells, when the phase of $\delta Z$ is arbitrarily set to zero. (b) Example of the data obtained by phase-field simulation for a single cell during three oscillation periods $\left(V_{p}=1 \mu \mathrm{m} / \mathrm{s}, G=24 \mathrm{~K} / \mathrm{cm}, \lambda=177 \mu \mathrm{m}\right): \delta Z$, green ; $\delta V$, blue $\Delta ; \rho$, red $\square ; C_{t}$, empty $\diamond$. (c) Cell shape evolution. The time labels correspond to the extrema and mean values of $\delta Z$ and $\rho$. The arrows at the tips represent an increasing or decreasing tip radius ( $\circ$ for unchanging). The vertical arrows in the middle of the cells represent $\delta V$. using a domain containing 1.5 cells, with no-flux boundary conditions in $y$ and "helical" boundary conditions in $x$ [26]. Simulations were performed using a box of $265 \mu \mathrm{m}$ in width, $156 \mu \mathrm{m}$ in thickness, and $3183 \mu \mathrm{m}$ in height.

These simulations lead to oscillating cells exhibiting hexagonal ordering where three subarrays oscillate with a phase shift of $\approx \pm \frac{2 \pi}{3}$, which is in good agreement with our experimental observations [24,25]. The evolution of the three geometrical parameters, tip radius, velocity, and position [Fig. 8(b)], lead to the phase shifts summarized in Table II and a simplified representation very similar to the experimental one. Additionally, the mean curvature radius found in phasefield simulations $(54 \pm 7 \mu \mathrm{m})$ is in very good agreement with the one found experimentally by interferometric analysis $(54 \pm 5 \mu \mathrm{m})$.

Thanks to these analyses, a scheme of the evolution of cell shape during a period of oscillation can be given in Fig. 8(c). While $\delta Z$ advances from its mean position, tip radius increases from near its minimum value. This tip radius increase continues well after $\delta Z$ has reached its maximum and has started to recede. The cell tip is bluntest when $\delta Z$ is decreasing and about to reach its mean position. The cell radius continues to decrease until after the cell reaches its minimum $\delta Z$. The existence of phase differences between the relevant characteristics of the cells is associated to the dynamics of oscillation; classical steady-state considerations such as, for example, that a faster tip is associated to a smaller tip radius are not valid in that dynamical asymptotic regime.
TABLE II. Phase relationships and amplitude ratios between the geometrical characteristic of oscillating tips: radius $\rho$, position $\delta z$, and velocity $\delta V$. Comparison between measured results in experiments and in 3D phase field simulation (PF) and theoretical model for both cases. The phase-relationships obtained by $\mathrm{PF}$ of $2 \lambda-O$ oscillating patterns [16] are also given.

\begin{tabular}{|c|c|c|c|c|c|}
\hline \multirow{2}{*}{$\begin{array}{l}\text { Phase shift } \\
\text { between }\end{array}$} & \multicolumn{2}{|c|}{ Experiments } & \multicolumn{2}{|c|}{$3 \mathrm{D} P F$} & \multirow{2}{*}{$\begin{array}{c}\mathrm{PF} \\
2 \lambda-O\end{array}$} \\
\hline & Meas. & Theor. & Meas. & Theor. & \\
\hline$\rho$ and $\delta z$ & $0.38 \pi$ & $0.29 \pi$ & $0.38 \pi$ & $0.34 \pi$ & $0.25 \pi$ \\
\hline$\rho$ and $\delta V$ & $0.86 \pi$ & $0.79 \pi$ & $0.86 \pi$ & $0.84 \pi$ & $0.75 \pi$ \\
\hline$\delta z$ and $\delta V$ & $0.52 \pi$ & $0.50 \pi$ & $0.48 \pi$ & $0.50 \pi$ & $0.50 \pi$ \\
\hline \multicolumn{6}{|c|}{ Amplitude ratios } \\
\hline$\frac{A_{v}}{A_{z}}\left(\mathrm{~s}^{-1}\right)$ & 0.0021 & 0.0020 & 0.0037 & 0.0038 & - \\
\hline$\frac{A_{\rho} / \rho_{0}}{A_{z}}\left(\mu \mathrm{m}^{-1}\right)$ & 0.013 & 0.002 & 0.032 & 0.005 & - \\
\hline
\end{tabular}

The experimental evolution scheme of Fig. 8(c) relating the cell tip shape and position (or undercooling) during oscillation is thus mandatory to propose an interpretation of the origin of oscillation.

These results can be compared to those of Georgelin and Pocheau [14] in thin samples. The scheme of Fig. 8(c) is quite similar to the images of Fig. 2 of Ref. [14]: the medium tip position of cells corresponds to either large or thin cells, the highest position of the tip corresponds to thick - but not thickest — cell and the lowest position of tip corresponds to thin — but not thinnest—cell. Georgelin and Pocheau [14] reported a phase difference of $0.56 \pi$ of tip position leading the cell width, 1.5 times larger than our measurement $(0.38 \pi)$, thus suggesting a faster dynamics of shape adjustment in our case. At this stage, it is not possible to relate this difference to the sample geometry or to growth parameters, as measurements are available for only one set of parameters in both cases.

\section{B. Analysis of solutal interactions driving oscillation}

Experimentally, the analysis of the oscillating patterns using interferometry made it possible to highlight and quantify the evolution of shape, velocity and position of the oscillating cells. They are associated to the evolution of the concentration field, which is not accessible experimentally but is clearly the key parameter to explain the coupling between neighboring cells. The advantage of phase field simulations is that, in addition to purely geometric characteristics of the cell tip, they provide the spatiotemporal evolution of the concentration field.

To evidence the very small variations of concentration associated to oscillation, Ghmadh et al. [16] have developed an original method of analysis based on the determination of local constitutional supercooling. The constitutional supercooling $\Delta C(\vec{r}, t)$ is defined as the difference between the equilibrium concentration of a liquid point, which is the liquidus concentration and the actual concentration $C(\vec{r}, t)$ :

$$
\Delta C(\vec{r}, t)=C_{L}(z, t)-C(\vec{r}, t) .
$$

It may also be interesting to compare these data to the 3D phase-field simulations conducted by Ghmadh et al. [16] to 


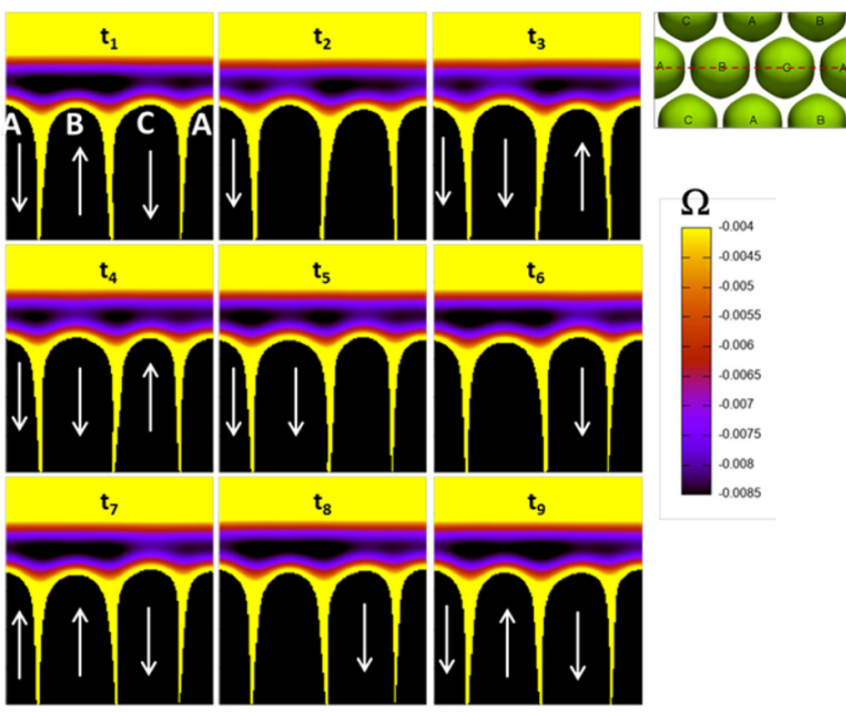

FIG. 9. Time evolution of the oscillation mode of a hexagonal array obtained numerically. Solid appears in black in the lower part; white arrows indicate the direction of the cells' motion; in the liquid phase, isosurfaces are drawn for the constitutional undercooling $\Omega$. One oscillation period corresponds to the time interval between $t_{1}$ and $t_{9}$. analyze $2 \lambda-O$ oscillating patterns obtained by Georgelin and Pocheau [14] in thin samples. The phase relationships are in very good agreement with the ones found experimentally as well as they are in the same range as those found by Ghmadh et al. [16]. Especially, in spite of slight differences of values, those phase shifts lead to a scheme of evolution similar to the one presented in Fig. 8(b).

A dimensionless undercooling $\Omega$ can then be defined as $\Omega=-\frac{k}{C_{0}} \Delta C$. Once the interface is destabilized, a zone of negative undercooling builds up in the liquid and $\Omega$ goes through a minimum at some distance ahead of the cell tips (in the solid, the undercooling is uniformly set to an arbitrary negative constant). $\Omega$ becomes more negative when the constitutional supercooling increases. We applied the same kind of analysis in this work, for the $\frac{2 \pi}{3}$-oscillating patterns.

Figure 9 represents $\Omega$ in the $x z$ plane that intersects the cell tips along the red dotted line in the top view. One can follow the evolution of the geometric parameters [Fig. 8(b)] by focusing attention on cell B of Fig. 9. Let us first consider the variation of tip velocity. At time $t_{2}, \delta V=0$ and it decreases until $t_{5}$. This decrease of velocity below the average tip velocity means that the vertical flux rejected at the tip decreases below its average value: the concentration ahead of the tip also decreases with a small time shift, producing the large black area that develops above the cell tip between $t_{4}$ and $t_{7}$. The increase of velocity between $t_{7}$ and $t_{9}$ above the average velocity induces the inverse phenomenon, with a resulting increase of concentration and the reduction of the black area above the tip. The same behavior shifted by $\frac{2 \pi}{3}$ is observed for the two other cells, A and B. Similar observations were done by Ghmadh et al. [16] in the $2 \lambda-O$ oscillating patterns, except that they had only two cells in phase opposition.

Whatever the system is, the question at this point is to identify the mechanism that enables us to counteract the variations

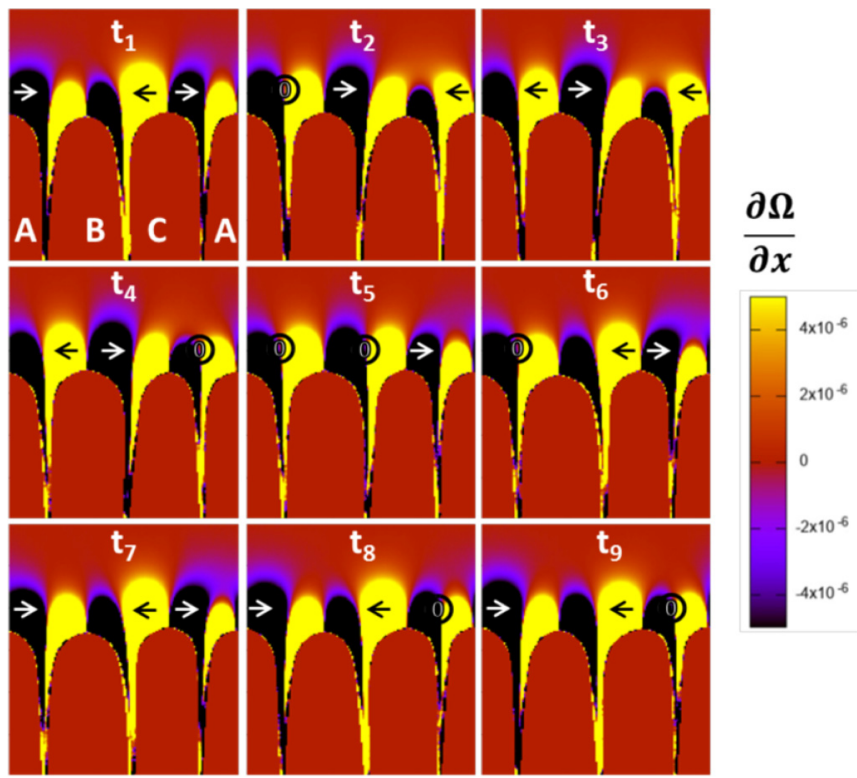

FIG. 10. Time evolution of the field $\frac{d \Omega}{d x}$ during one oscillation period $\left(t_{1}\right.$ to $\left.t_{9}\right)$. The arrows indicate the orientations of the horizontal solute currents that converge towards the neighbor cell; $\circ$ denotes zero flow. At the bottom and the top of the frames, the zero current areas, respectively, represent the solid and the far-field liquid.

of velocity and concentration to go back to average values: such a mechanism is necessary to explain why, for example, a cell would not continue to recoil and eventually be eliminated when the velocity decreases. As described in Ref. [16], it is possible to evidence horizontal solute fluxes, by representing $\frac{d \Omega}{d x}$ (in the solid, $\frac{d \Omega}{d x}=0$ ): Fig. 10 shows the time evolution of $\frac{d \Omega}{d x}$ over an oscillation period. On the same basis used for the $2 \lambda-O$ oscillating patterns [16], it is possible to explain the cycle of oscillation, taking into account horizontal solute fluxes coming from neighboring cells. When the concentration is minimal above the cell tip B (large black area above the cells), the concentration above cell tips $\mathrm{A}$ and $\mathrm{C}$ is higher than above $\mathrm{B}$ : the horizontal solute currents coming from the cells $\mathrm{A}$ and $\mathrm{C}$ converge, which induce a melting of the tip and sides of cell $\mathrm{B}$. The tip radius of $\mathrm{B}$ decreases to reach its minimum, thus inducing an increase of diffusion currents at the tip and consequently an increase of the tip velocity and of the concentration above B. Compared to the $2 \lambda-O$ oscillation mode, the main difference evidenced in Fig. 10 is the asymmetry of the horizontal fluxes coming to or from B. This probably results from the phase shift of $\pm \frac{2 \pi}{3}$ between the three subpatterns, and this provokes an asymmetry of the cell shape clearly visible in Fig. 10.

The two mechanisms that modify the solute concentration (and thus the undercooling) above the tip are identified: a vertical one and a horizontal one resulting from interaction with neighbors. The coupling of these two mechanisms involved in the oscillation fixes the phase shifts between the oscillation of the different cell tip characteristics, since one of these mechanisms is based on neighbor interaction. We may suspect that the pattern organization and the coherence and synchronization mode of oscillation affect those phase shifts. 


\section{Theoretical analysis of the cell tip dynamics}

Let $\delta Z, \delta V, \delta \rho, \delta C_{t}$, respectively, denote the oscillating components of the tip position, velocity, curvature radius and interfacial liquid solute concentration at the cell tip. Sine variations are considered which will be treated as complex numbers for easier calculations: $\tilde{\delta} \rho=A_{\rho} e^{i(\omega t)}, \tilde{\delta} v=$ $A_{v} e^{i\left(\omega t+\phi_{v}\right)}, \tilde{\delta} z=A_{z} e^{i\left(\omega t+\phi_{z}\right)}, \tilde{\delta} C_{t}=A_{c} e^{i\left(\omega t+\phi_{c}\right)}$. Since the velocity is given by the temporal derivative of the tip position, $V(t)=\dot{z}(t)$, we have

$$
\frac{A_{v}}{A_{z}}=\omega \text { and } \phi_{v}=\phi_{z}+\frac{\pi}{2} .
$$

Moreover, local equilibrium at the interface implies that

$$
\delta C_{t}=\frac{G \delta z}{m} \text { with } m<0
$$

so that

$$
\frac{A_{c}}{A_{z}}=\frac{G}{|m|} \text { and } \phi_{c}=\phi_{z}+\pi .
$$

The existence of local equilibrium at the interface implies the solute diffusion time in the liquid to be much faster than the oscillation period. As previously mentioned, the mean oscillation period is equal to $52 \pm 6 \mathrm{~min}$. The solute diffusion time can be estimated by the ratio between the square of the diffusion length and the solute diffusion coefficient in the liquid $D_{L}$ (Table I). In our case, a relevant diffusion length would be the primary spacing $(288 \pm 42 \mu \mathrm{m})$. So, the solute diffusion time may be estimated as $5.1 \mathrm{~min}$, which is $\approx 10$ times smaller than $\tau_{\text {osc }}$. We can then consider that the conditions for local equilibrium are respected. An additional selection criterion that defines the operating conditions at the cell tip and relates the tip radius to the other oscillating tip characteristics is imposed to determine the remaining phase shift. Even if it is more adapted to the dendritic regime, we consider that the tip radius is selected through the solvability approach, taking into account capillarity $[48,49]$ :

$$
\rho^{2}\left(m G_{c}-G\right)=\frac{\Gamma}{\sigma^{*}} \Rightarrow 2 \rho_{0} \delta \rho\left(m G_{c, 0}-G\right)+\rho_{0}^{2} m \delta G_{c}=0,
$$

where $G_{c}$ is the concentration gradient at the tip, $\Gamma$ the GibbsThomson coefficient, and $\sigma^{*}$ the stability constant. $\rho_{0}$ and $G_{c, 0}$ are the average values of $\rho$ and $G_{c}$, respectively.

The concentration gradient at the tip may be defined by the solute flow balance:

$$
\begin{aligned}
G_{c}(t) & =\frac{k-1}{D_{L}} C_{t}(t) V(t) \Rightarrow \\
\delta G_{c}(t) & =\frac{k-1}{D_{L}}\left(V_{p} \delta C_{t}+C_{t, 0} \delta V\right), \text { and } \\
G_{C, 0} & =\frac{k-1}{D_{L}} C_{t, 0} V_{p},
\end{aligned}
$$

where $C_{t, 0}$ is the average value of $C_{t}$ and the average value of the velocity is equivalent to the pulling velocity, $V_{p}$. Combining Eqs. (14) and (15):

$$
2 \frac{\delta \rho}{\rho_{0}}=-B\left(\frac{\delta C_{t}}{C_{t, 0}}+\frac{\delta V_{t}}{V_{p}}\right)
$$

with $\frac{1}{B}=1-\frac{G D_{L}}{m V_{p} C_{t, 0}(k-1)}>0$.
Using the complex notation previously defined, and the results of Eqs. (11)-(13), one gets

$$
\begin{aligned}
2 \frac{A_{\rho}}{\rho_{0}} & =e^{-i \pi} B\left(\frac{A_{c}}{C_{t, 0}} e^{i \phi_{c}}+\frac{A_{v}}{V_{p}} e^{i \phi_{v}}\right) \\
& =B e^{i \phi_{z}}\left(\frac{A_{c}}{C_{t, 0}}-i \frac{A_{v}}{V_{p}}\right),
\end{aligned}
$$

which is equivalent to

$$
\frac{A_{\rho}}{A_{z}}=B \frac{\rho_{0}}{2} \sqrt{\left(\frac{G}{|m| C_{t, 0}}\right)^{2}+\left(\frac{\omega}{V_{p}}\right)^{2}}
$$

$$
\text { and } \tan \left(\phi_{z}\right)=\frac{C_{t, 0}}{V_{p}} \frac{\omega|m|}{G} .
$$

The average tip concentration $C_{t, 0}$ can be calculated using $\quad 692$ the undercooling $\Delta_{K P}$ defined in the Karma-Pelcé (KP) model $[50,51]:$

$$
\Delta_{K P}=\frac{T_{L}-T_{t, 0}}{T_{L}-T_{S}}=\frac{f_{s, 0} k+\left(1-f_{s, 0}\right) \frac{D_{L}}{V_{P} T_{T}}}{1-f_{s, 0}(1-k)},
$$

where $T_{t, 0}$ is the average tip temperature, $T_{L}$ and $T_{S}$ are the liquidus and solidus temperatures for the composition $C_{0}$, respectively. $l_{T}$ is the thermal length defined as $\frac{m C_{0}(k-1)}{k G} . f_{s}$ is defined as the solid fraction in a region behind the tip where the cell width can be assumed constant; $f_{s, 0}$ is the average value of $f_{s}$. It should be pointed out that Eq. (19) reduces to the well-known Bower-Brody-Flemings model [52] when $f_{s, 0}$ vanishes. From Eq. (19), we get

$$
C_{t, 0}=C_{0}\left(1-\frac{k-1}{k} \Delta_{K P}\right) \text {. }
$$

Equations (11)-(18) are used to calculate all the phase shifts and oscillation amplitudes that define the oscillating cell dynamics, to compare them with experimental and numerical simulation results. In addition to the experimental control parameters and to the characteristics of the alloy, the tip concentration is required for the calculations. For phasefield simulations, the tip undercooling is directly provided and converted in tip concentration using Eq. (20). The tip concentration evolution is represented by the dark curve in Fig. 8(b), for $V_{p}=1 \mu \mathrm{m} / \mathrm{s}, G=24 \mathrm{~K} / \mathrm{cm}$ and $\lambda=177 \mu \mathrm{m}$, and a sinusoidal fit leads to $C_{t, 0}=0.85 \mathrm{wt} \%$ and $A_{c}=0.01$. The analysis of the sinusoidal fits of $C_{t}$ and $\delta z$ is in accordance with Eq. (13). In experiments, the tip concentration is not available, but we can estimate the solid fraction $f_{s}$ deduced from the measurements of the visible cell surface $S$ and use Eqs. (19) and (20) to characterize the experimental tip concentration. Considering an ideal hexagonal pattern, $f_{s}$ can be evaluated as the ratio between $S$ and the area of a hexagon with an apothem equal to the half of the primary spacing. This calculation leads to $f_{s, 0} \approx 0.60$ and $A_{f_{s}}=0.01$, and $C_{t, 0}=0.89 \mathrm{wt} \%$ and $A_{c}=0.02$. However, this determination of the solid fraction is relatively imprecise as we cannot be sure that the cell visible surface $S$ exactly fits the cell size (it may be underestimated depending on optical visibility) and the calculation of the fraction is based on the hypothesis of a perfect hexagonal pattern, which is not experimentally the case. The experimental and simulation values of $C_{t, 0}$ are nonetheless very close. 
The values of phase shifts are gathered in Table II: the theoretical values are compared to both experimental and numerical values. Using the numerical oscillation period $T=$ $27 \mathrm{~min}$ and the estimated average tip concentration $0.85 \mathrm{wt} \%$, the theoretical phase shift of $z$ is calculated $\phi_{z}=0.34 \pi$; it is quite similar to its value directly measured in PF and to the experimental value (both $0.38 \pi$ ). With the experimental parameters $\left(T=52 \mathrm{~min}\right.$ and $\left.C_{t, 0}=0.89 \mathrm{wt} \%\right)$, the theoretical phase shift of $Z$ is equal to $\phi_{z}=0.29 \pi$, significantly lower than the experimentally measured value of $0.38 \pi$ as well. It appears that this phase shift is very sensitive to the solid fraction $f_{s}$ : a $f_{s, 0} \approx 0.90$ would be necessary to get a perfect agreement between theory and experiment. The other phase shifts are deduced from the derivative relationship between $V$ and $z$, and the phase shifts between experiments, or simulation, and the theoretical calculation are quite consistent.

Regarding the ratio of oscillation amplitudes, the theoretical $\frac{A_{v}}{A_{z}}=0.0020 \mathrm{~s}^{-1}$ is very close to the experimental one $\left(0.0021 \mathrm{~s}^{-1}\right)$, and, similarly, between the theoretical $0.0038 \mathrm{~s}^{-1}$ and the phase-field simulation $0.0037 \mathrm{~s}^{-1}$. It should be pointed out that this agreement is trivially expected since the ratio $\frac{A_{v}}{A_{z}}$ is fixed by the oscillation period following Eq. (11) and the period is accurately determined in both experiment and simulation. Relation (18) is used to calculate the theoretical value of $\frac{A_{\rho} / \rho_{0}}{A_{z}}$. For the phase-field simulation working parameters, its value is $0.005 \mu \mathrm{m}^{-1}$, which is almost six times lower than the one estimated using the directly measured amplitudes of oscillation $\left(0.032 \mu \mathrm{m}^{-1}\right)$. Experimentally, the variations of $\frac{A_{\rho}}{\rho_{0}}$ as a function of $A_{z}$ lead to a slope of $0.013 \mu \mathrm{m}^{-1}$, and the estimated value through Eq. (18) is equal to $0.002 \mu \mathrm{m}^{-1}$, also six times lower. We may suggest two different hypotheses for the discrepancy observed in the ratio of oscillation amplitudes $\frac{A_{\rho} / \rho_{0}}{A_{z}}$.

First, we recall that Eq. (14) was developed for a dendritic regime. In that case, tips can be approximated as isolated parabolic tips, with almost no overlap of the concentration field. It is not exact in case of cells and especially of oscillating cells for which the solute fluxes between neighboring cells are critical during the oscillation cycle. To see how the equations would be modified by using a specifically cellular equation, we can replace Eq. (14) by an equation developed in the theoretical framework of the analogy between cells and Saffman-Taylor (ST) viscous fingers [50,53]. Its use is neither fully justified because it applies theoretically within the limit of small cell tip Péclet number $\left(\mathrm{Pe}=\frac{\lambda V_{p}}{D_{L}} \ll 1\right.$, where $\lambda$ is the primary spacing), which is not our case $(\mathrm{Pe} \approx 1)$. The cell shape is determined by the relative width $\Lambda$ of the cell, which corresponds to the ratio of the cell width with the primary spacing. In analogy with ST solutions, the selection of $\Lambda$ proceeds through a capillary function $F_{\Lambda}(\Lambda)$ that depends on a parameter that quantifies surface tension effects [54]:

$$
\rho^{2}\left(m G_{c}-G\right)=\Gamma F_{\Lambda}(\Lambda) .
$$

We see that, if the term $F_{\Lambda}(\Lambda)$ is set to a specific constant, we go back to Eq. (14) developed for a dendritic regime $[48,49]$. Equations (14) and (21) therefore describe two limit domains, our case being between them. The ST finger shape basically contains two shape parameters, $\frac{\rho}{\lambda}$ and $\Lambda$, related to each other, so that only one of these variables is required to describe the shape: $F_{\Lambda}(\Lambda)=F_{\Lambda}\left(\frac{\rho}{\lambda}\right)$. In the oscillating experiments, the primary spacing attains a steady-state value: the capillary function is then supposed to vary only due to tip radius oscillations and we can switch from $F_{\Lambda}\left(\frac{\rho}{\lambda}\right)$ to $F_{\rho}(\rho)$. After differentiation, we get

$$
2 \rho_{0} \delta \rho\left(m G_{c, 0}-G\right)+\rho_{0}^{2} m \delta G_{c}=\Gamma \delta F_{\rho}(\rho) .
$$

In a 3D pattern, we can consider that the relative width $\Lambda$ is similar to the solid fraction $f_{S} \propto \frac{S}{\lambda^{2}}$. As the apparent surface $S$ oscillates in phase with the tip radius, it thus follows that the solid fraction, the relative width, the tip radius and the capillary function oscillations are all in phase. We then define the complex number for $\delta F_{\rho}$, with the tip radius fixing the origin of phases: $\tilde{\delta} F_{\rho}=A_{F} e^{i(\omega t)}$. The calculation is carried out in the same way and with the same assumptions as previously done, and Eqs. (17) and (18) can be replaced by

$$
2 \frac{A_{\rho}}{\rho_{0}}-\frac{A_{F}}{F_{0}}=B e^{i \phi_{z}}\left(\frac{A_{c}}{C_{t, 0}}-i \frac{A_{v}}{V_{p}}\right),
$$

where $F_{0}$ is the average value of $F_{\Lambda}(\Lambda)$ defined as $\frac{\rho_{0}^{2}\left(m G_{c, 0}-G\right)}{\Gamma}$. Equation (23) is equivalent to

$$
\begin{aligned}
\frac{A_{\rho}}{A_{z}} & =\frac{\rho_{0}}{2 F_{0}} \frac{A_{F}}{A_{z}}+B \frac{\rho_{0}}{2} \sqrt{\left(\frac{G}{|m| C_{t, 0}}\right)^{2}+\left(\frac{\omega}{V_{p}}\right)^{2}} \\
\text { and } \tan \left(\phi_{z}\right) & =\frac{C_{t, 0}}{V_{p}} \frac{\omega|m|}{G} .
\end{aligned}
$$

We note that the theoretical phase shift of $z$ is not affected by the modification, so that the small differences between measured and theoretical phase shifts do not come from the incorrect choice of the equation for the operating point. However, for amplitudes, $\frac{A_{\rho}}{A_{z}}$ is increased by the capillary function oscillation $\frac{A_{F}}{F_{0}}$ when compared to the previous expression. It was previously observed that Eq. (18) leads to a large underestimation of $\frac{A_{\rho}}{A_{z}}$ when compared to the measured value: the correct description of the operating point could partly explain the difference. Applying the limit case of ST analogy described by Eq. (24), we can evaluate a relative oscillation amplitude for the capillary function $\frac{A_{F}}{F_{0}}: 24 \%$ and $28 \%$ with, respectively, $\mathrm{PF}$ and experimental parameters. Those values are almost twice the relative oscillation amplitude of tip radius; inversely, if a ratio $\frac{A_{F}}{F_{0}}$ similar to the relative oscillation amplitude of tip radius is used, the discrepancy between theoretical and measured values of $\frac{A_{\rho} / \rho_{0}}{A_{z}}$ decreases from a ratio of 6 to 1.4. A correct description of the operating point would probably lead to an intermediate situation between the use of Eqs. (14) and (21), with still an underestimation of the tip radius oscillation, but largely reduced compared to the use of Eq. (14). We also can postulate that the phase-shift expression would not be modified.

The second element that could explain the discrepancy observed in the ratio of oscillation amplitudes $\frac{A_{\rho} / \rho_{0}}{A_{z}}$ is the fact that this theoretical analysis does not take into account the solute fluxes that come from neighboring cells and that are essential in the oscillating mechanism. These fluxes modify the concentration field and the resulting tip concentration 
and gradient. It is difficult to include these fluxes in the analysis but they also partly affect the oscillation amplitudes ratio and more importantly, necessarily affect the phase shift: as a matter of fact, the dynamics of these fluxes strongly depends on the pattern organization and of the type of oscillation coherence. In the $\frac{2 \pi}{3}$ type or $2 \lambda-O$ oscillations, the neighboring fluxes have different dynamics: we note that in spatially extended PF and experimental cases, the underlying pattern order is similar (hexagonal type) and the measured phase shifts have the same value $\left(\phi_{z}=0.38 \pi\right)$ whereas in simulation of $2 \lambda-O$, the phase shift is smaller $\left(\phi_{z}=0.25 \pi\right)$.

As a conclusion, the basic solidification equations can be a good starting point to describe the oscillation dynamics. A qualitative analysis suggests that the oscillation amplitudes would be better evaluated mainly by a more adequate description of the operating point. The neighboring solute fluxes, and therefore the underlying pattern order, mainly control the phase shifts of the different oscillating parameters.

\section{CONCLUSIONS}

Experiments under low-gravity conditions were carried out in the directional solidification insert (DSI) of the DECLIC facility installed on board the International Space Station as part of a joint research program between CNES and NASA. The use of an organic transparent alloy in a large cylindrical crucible enabled the real-time and in situ observation of spatially extended patterns, and microgravity enabled growth in a diffusive transport mode. These exceptional conditions allowed us to observe, for the first time, the dynamics of extended oscillating cellular 3D patterns. In some previous articles [24-26], we characterized the oscillations through the study of the periodic variation of the apparent area of cells in the $x y$ plane-which is in fact associated to a tip radius oscillation-using top-view interface observation. However, oscillating cells also display variations of the tip position and velocity in the vertical $Z$ direction. In the present work, the interferometric data available in DECLIC-DSI were used to reconstruct cell shape and measure oscillations of the cell tip position $\delta Z$ and growth velocity $\delta V$, in order to completely quantify the dynamics of oscillating cells. Moreover, the interface shape was reconstructed for each cell which allowed measuring the radius of curvature and its oscillations. The method of interferometric images analysis, for which it is not trivial to reach the highest resolution in spite of noise and nonuniform contrast conditions, was described. The individual cell shapes were then reconstructed with a $\rho$ resolution of $\approx 3 \mu \mathrm{m}$, and the evolution of cell growth velocity with a resolution of a few $\mathrm{nm} / \mathrm{s}$. Comparison with the cell apparent surfaces showed that $S$ and $\rho$ are correlated and in phase. A value of $0.86 \pi$ was measured for the phase shift between $\rho$ and $\delta V$, showing that cell tip radius and cell velocity are part of a rather complex dynamic of advance and bulging. These analyses allowed us to propose a scheme of the evolution of the cell shape during one period of oscillation. While $Z$ advances from its mean position, $\rho$ increases from its minimum value and it continues well after $Z$ has reached its maximum position and started to recede. The cell tip is bluntest when $Z$ is decreasing and about to reach its mean position.

The oscillations of tip shape parameters and tip position were correlated to the evolution of the concentration field by using phase-field simulations that, additionally to the purely geometric parameters of the cell tip, gave the spatiotemporal evolution of the concentration field. Phase-field simulations were performed to compare with the experimental results on extended arrays. The phase shifts of the geometrical parameters obtained experimentally and in simulation proved perfectly comparable. Due to simulation results, two mechanisms modifying the solute concentration above the tip were identified, a vertical one and a horizontal one resulting from the interaction with neighbor cells. Similarly to what was recently evidenced for $2 \lambda-O$ oscillations in thin samples [16], the dynamics of these two mechanisms was found to determine the phase shifts between the oscillations of the different cell tip characteristics.

A theoretical modeling based on classical equations of solidification enables us to analyze the oscillation characteristics of the different tip parameters. The oscillation amplitude of the tip radius estimated using the theoretical model is significantly smaller than the measured one. Two limit cases are used in the model to describe the tip operating point and relate the tip radius to the undercooling, corresponding to small and large Péclet numbers. Even if our situation lies between these limit cases, this approach enabled us to evidence the importance of the tip-shape selection on the oscillation amplitudes; it also demonstrated that phase shifts between the different oscillating quantities are not affected by the tip-shape selection. The theoretical phase shifts are rather consistent with experimental measurements as well as phase-field numerical simulations. However, we expect these phase shifts to be influenced by the neighboring solute fluxes-linked to the underlying pattern order-which are not included in the model. Thus, 3D phase-field analyses of square patterns, with two subpatterns in phase opposition, would also be interesting to compare to the hexagonal configuration analyzed in this work.

\section{ACKNOWLEDGMENTS}

We would like to thank Samuel Quiret for his help to build the database of image analysis procedures. This research was achieved thanks to the support of CNES through the MISOL3D project (MIcrostructures de SOLidification 3D), National Aeronautics and Space Administration (NASA) through Grants No. NNX12AK54G and 80NSSC19K0135, and Région PACA through the ENEMS project (Etude Numérique et Expérimentale des Microstructures de Solidification).
[1] M. Cross and H. Greenside, Pattern Formation and Dynamics in Nonequilibrium Systems (Cambridge University Press, New York, 2009).

[2] J. A. Dantzig and M. Rappaz, Solidification (EPFL Press, Lausanne, 2009).
[3] W. Kurz and D. J. Fisher, Fundamentals of Solidification (Trans Tech Pub, Zurich, 1998).

[4] P. Pelcé, Théorie Des Formes De Croissance (EDP Sciences/CNRS Éditions, Paris, 2012). 
[5] W. A. Tiller, K. A. Jackson, J. W. Rutter, and B. Chalmers, Acta Mater. 1, 428 (1953).

[6] W. Mullins and R. Sekerka, J. Appl. Phys. 35, 444 (1964).

[7] K. Kassner, C. Misbah, H. Müller-Krumbhaar, and A. Valance, Phys. Rev. E 49, 5477 (1994).

[8] C. Misbah and A. Valance, Phys. Rev. E 49, 166 (1994).

[9] A. J. Simon, J. Bechhoefer, and A. Libchaber, Phys. Rev. Lett. 61, 2574 (1988).

[10] W. Losert, D. A. Stillman, H. Z. Cummins, P. Kopczynski, W. J. Rappel, and A. Karma, Phys. Rev. E 58, 7492 (1998).

[11] P. Coullet and G. Iooss, Phys. Rev. Lett. 64, 866 (1990).

[12] B. Grossmann, K. R. Elder, M. Grant, and J. M. Kosterlitz, Phys. Rev. Lett. 71, 3323 (1993).

[13] P. E. Cladis, J. T. Gleeson, P. L. Finn, and H. R. Brand, Phys. Rev. Lett. 23, 3239 (1991).

[14] M. Georgelin and A. Pocheau, Phys. Rev. Lett. 79, 2698 (1997).

[15] P. Kopczyński, W. J. Rappel, and A. Karma, Phys. Rev. Lett. 77, 3387 (1996).

[16] J. Ghmadh, J.-M. Debierre, M. Georgelin, R. Guérin, and A. Pocheau, Phys. Rev. E 98, 052802 (2018).

[17] A. Karma and A. Sarkissian, Metall. Mater. Trans. A 27, 635 (1996).

[18] M. Ginibre, S. Akamatsu, and G. Faivre, Phys. Rev. E 56, 780 (1997).

[19] S. Akamatsu, S. Bottin-Rousseau, M. Perrut, G. Faivre, V. T. Witusiewicz, and L. Sturz, J. Cryst. Growth 299, 418 (2007).

[20] M. Zimmermann, A. Karma, and M. Carrard, Phys. Rev. B 42, 833 (1990).

[21] S. C. Gill and W. Kurz, Acta Metall. Mater. 41, 3563 (1993).

[22] K. Kassner, J.-M. Debierre, B. Billia, N. Noël, and H. Jamgotchian, Phys. Rev. E 57, 780 (1998).

[23] M. Plapp and M. Dejmek, Europhys. Lett. 65, 276 (2004).

[24] N. Bergeon, D. Tourret, L. Chen, J.-M. Debierre, R. Guérin, R. Ramirez, B. Billia, A. Karma, and R. Trivedi, Phys. Rev. Lett. 110, 226102 (2013).

[25] J. Pereda, F. L. Mota, L. Chen, B. Billia, D. Tourret, Y. Song, J.M. Debierre, R. Guérin, A. Karma, R. Trivedi, and N. Bergeon, Phys. Rev. E 95, 012803 (2017).

[26] D. Tourret, J.-M. Debierre, Y. Song, F. L. Mota, N. Bergeon, R. Guérin, R. Trivedi, B. Billia, and A. Karma, Phys. Rev. E 92, 042401 (2015).

[27] N. Bergeon, R. Trivedi, B. Billia, B. Echebarria, A. Karma, S. Liu, C. Weiss, and N. Mangelinck, Adv. Space Res. 36, 80 (2005).

[28] N. Bergeon, A. Ramirez, L. Chen, B. Billia, J. Gu, and R. Trivedi, J. Mater. Sci. 46, 6191 (2011).

[29] R. Marcout, G. Raymond, B. Martin, G. Cambon, B. Zappoli, F. Duclos, S. Barde, D. Beysens, Y. Garrabos, C. Lecoutre, B. Billia, N. Bergeon, and N. Mangelinck, in 57th International Astronautical Congress, AIAA Meeting Paper, Valencia, IAC06-A2.5.02 (2006).

[30] G. Pont, S. Barde, B. Zappoli, F. Duclos, Y. Garrabos, C. Lecoutre, D. Beysens, B. Billia, N. Bergeon, N. Mangelinck, R. Marcout, D. Blonde, in 60th International Astronautical
Congress, International Astronautical Federation, Daejeon, IAC-09-A2.6.4 (2009).

[31] N. Bergeon, C. Weiss, N. Mangelinck-Noel, and B. Billia, Trans. Indian Inst. Met. 62, 455 (2009).

[32] H. Jamgotchian, N. Bergeon, D. Benielli, P. Voge, and B. Billia, J. Microsc.-Oxford 203, 119 (2001).

[33] B. Echebarria, R. Folch, A. Karma, and M. Plapp, Phys. Rev. E 70, 061604 (2004).

[34] National Institute of Standards and Technology (NIST): NIST Chemistry Webbook (NIST, Gaithersburg, MD, 2011).

[35] K. Somboonsuk, J. T. Mason, and R. Trivedi, Metall. Trans. A 15, 967 (1984).

[36] J. C. LaCombe, J. L. Oudemool, M. B. Koss, L. T. Bushnell, and M. E. Glicksman, J. Cryst. Growth 173, 167 (1997).

[37] Q. Li and C. Beckermann, J. Cryst. Growth 236, 482 (2002).

[38] J. Teng and S. Liu, J. Cryst. Growth 290, 248 (2006).

[39] F. L. Mota, L. M. Fabietti, N. Bergeon, L. L. Strutzenberg, A. Karma, B. Billia, and R. Trivedi, J. Cryst. Growth 447, 31 (2016).

[40] C. Weiss, N. Bergeon, N. Mangelinck-Noel, and B. Billia, Phys. Rev. E 79, 011605 (2009).

[41] F. L. Mota, N. Bergeon, D. Tourret, A. Karm, R. Trivedi, and B. Billia, Acta Mater. 85, 362 (2015).

[42] M. Kurz, A. Pusztai, and G. Muller, J. Cryst. Growth 198, 101 (1999).

[43] Y. Inatomi, H. Miyashita, E. Sato, K. Kuribayashi, K. Itonaga, and T. Motegi, J. Cryst. Growth 130, 85 (1993).

[44] R. M. Macfarlane, E. Courtens, and T. Bischofberger, Mol. Cryst. Liq. Cryst. 35, 27 (1976).

[45] L. Chen, Dynamical microstructure formation in 3D directional solidification of transparent model alloys: In situ characterization in DECLIC-DSI under diffusion transport in microgravity, Matières condensée et Nanosciences, Ph.D. thesis, Aix-Marseille Université, 2013.

[46] Z. Wang and B. Han, Opt. Lett. 29, 1671 (2004).

[47] See Supplemental Material at http://link.aps.org/supplemental/ 10.1103/PhysRevE.xx.xxxxxx for the evolution of the single cell number 50 (located at the interface point with the coordinates $x=418 p x$ and $y=209 p x$ ) during three interferometric and three direct observation sequences. The axis in the movie is scaled in pixels. During the interferometric sequences, interference fringes can be considered as level curves of the solid-liquid interface, allowing the obtaining of the radius of curvature evolution and cell position deviation. The direct observation yields the evolution of the visible cell surface.

[48] R. Trivedi and W. Kurz, Int. Mater. Rev. 39, 49 (1994).

[49] W. Kurz and R. Trivedi, Acta Metall. Mater. 38, 1 (1990).

[50] A. Karma and P. Pelcé, Phys. Rev. A 39, 4162 (1989).

[51] S. Gurevich, A. Karma, M. Plapp, and R. Trivedi, Phys. Rev. E 81, 1603 (2010).

[52] T. F. Bower, H. D. Brody, and M. C. Flemings, Trans. Metall. Soc. AIME 236, 624 (1966).

[53] P. Pelcé and A. Pumir, J. Cryst. Growth 73, 337 (1985).

[54] B. Billia and R. Trivedi, in Handbook of Crystal Growth, Vol. 1, edited by D. T. J. Hurle (North-Holland, Bristol, 1993), p. 899. 